\title{
Bariatric Surgery in Cirrhotic Patients: Is It Safe?
}

\section{Hafsa Younus $^{1}$ (D) Amit Sharma ${ }^{1} \cdot$ Rosa Miquel $^{1} \cdot$ Alberto Quaglia $^{1} \cdot$ Subba Rao Kanchustambam ${ }^{1}$. Kirstin A Carswell ${ }^{1}$. Ameet G. Patel ${ }^{2}$}

Published online: 18 December 2019

(C) The Author(s) 2019

\begin{abstract}
Introduction Ten percent of cirrhotic patients are known to have a high risk of postoperative complications. Ninety percent of bariatric patients suffer from non-alcoholic fatty liver disease (NAFLD), and 50\% of them may develop non-alcoholic steatohepatitis (NASH) which can progress to cirrhosis. The aim of this study was to assess whether the presence of cirrhosis at the time of bariatric surgery is associated with an increased rate and severity of short- and long-term cirrhotic complications.

Methods A cohort of 110 bariatric patients, between May 2003 and February 2018, who had undergone liver biopsy at the time of bariatric surgery were reassessed for histological outcome and divided into two groups based on the presence $(\mathrm{C}, n=26)$ or absence (NC, $n=84)$ of cirrhosis. The NC group consisted of NASH $(n=49)$, NAFLD $(n=24)$ and non-NAFLD $(n=11)$ liver histology. Medical notes were retrospectively assessed for patient characteristics, development of 30-day postoperative complications, severity of complications (Clavien-Dindo (CD) classification) and length of stay. The $\mathrm{C}$ group was further assessed for long-term cirrhosis-related outcomes.

Results The C group was older (52 years vs 43 years) and had lower BMI ( $46 \mathrm{~kg} / \mathrm{m}^{2}$ vs $52 \mathrm{~kg} / \mathrm{m}^{2}$ ) and weight (126 kg vs $145 \mathrm{~kg}$ ) compared to the $\mathrm{NC}$ group $(p<0.05)$. The $\mathrm{C}$ group had significantly higher overall complication rate $(10 / 26 \mathrm{vs} 14 / 84, p<0.05)$ and severity of complications (CD class $\geq \mathrm{III}, 12 \%$ vs $7 \%, p<0.05$ ) when compared to the NC group. The length of stay was similar between the two groups ( 5 days vs 4 days). The $\mathrm{C}$ group had significant improvement in model end-stage liver disease scores ( 7 vs $6, p<0.01)$ with median follow-up of 4.5 years (range $2-11$ years). There were no long-term cirrhosis-related complications or mortality in our studied cohort $(0 / 26)$.

Conclusion Bariatric surgery in cirrhotic patients has a higher risk of immediate postoperative complications. Long-term cirrhosis-related complications or mortality was not increased in this small cohort. Preoperative identification of liver cirrhosis may be useful for risk stratification, optimisation and informed consent. Bariatric surgery in well-compensated cirrhotic patients may be used as an aid to improve long-term outcome.
\end{abstract}

Keywords Bariatric surgery $\cdot$ Cirrhosis $\cdot$ NAFLD $\cdot$ Adults $\cdot$ Complications

Ameet G. Patel

ameet.1.patel@kcl.ac.uk

Hafsa Younus

hafsayounus@nhs.net

Amit Sharma

amitsharma1538@gmail.com

Rosa Miquel

rosa.miquel@nhs.net

Alberto Quaglia

Alberto.quaglia@nhs.net
Subba Rao Kanchustambam subba.kanchustambam@nhs.net

Kirstin A Carswell

kirstincarswell@nhs.net

King's College Hospital, London, UK

2 Department of Minimal Access Surgery, King's College Hospital NHS Foundation Trust, Denmark Hill, London SE5 9RS, UK 


\section{Introduction}

Mild to moderate liver steatosis shows a predilection for centrilobular distribution, progressing to diffuse fatty accumulation eventually resulting in non-alcoholic steatohepatitis (NASH) or fibrosis [1]. The presence of hepatic steatosis makes liver susceptible to cytokine-mediated injury resulting in steatohepatitis and/or fibrosis [2-7]. Fibrosis or cirrhosis represents the final common end point of almost all chronic liver diseases including NASH.

In obesity, an enhanced rate of lipolysis leads to increased availability of free fatty acids responsible for an exaggerated synthesis and deposition of triglycerides in hepatocytes $[8,9]$. Ninety percent of patients with morbid obesity suffer from non-alcoholic fatty liver disease (NAFLD), eventually resulting in NASH in $50 \%$ of patients undergoing bariatric surgery $[1,10,11]$.

Cirrhotic patients are known to have increased perioperative risk for both abdominal and non-abdominal surgery [12]. They have an increased risk of mortality from liver and renal failure, and impaired coagulation may result in the sequelae of postoperative bleeding [10]. Once cirrhosis is established, patients can progress from an asymptomatic compensated stage to a symptomatic decompensated stage. The latter maybe marked by the development of clinical complications of portal hypertension and liver failure. Although the risk of mortality after bariatric surgery is $0.9 \%$ in the compensated stage, it increases up to $16 \%$ upon decompensation as compared to only $0.3 \%$ in non-cirrhotic patients [13].

The aim of this study was to assess whether the presence of cirrhosis at the time of bariatric surgery leads to an increased rate, severity of short-term complications and length of stay compared to non-cirrhotic patients. We also studied the effect of bariatric surgery on long-term cirrhosis-related complications.

\section{Methods}

\section{Study Design and Participants}

Between May 2003 and February 2018, a cohort of 1676 morbidly obese patients underwent bariatric surgery under a single surgeon. At the time of surgery, all patients who had incidental macroscopic appearance of liver cirrhosis underwent a liver biopsy $(n=26)$. The incidence of unexpected cirrhosis in this cohort was $1.5 \%$ (26/1676). Non-cirrhotic liver biopsies were taken on random patients from this cohort of 1676 patients as part of other studies $(n=90)$. Patients who underwent intraoperative liver biopsies were assessed for suitability. Of a total of 116 patients, 110 patients were included in the study. Patients who had other known aetiological factors for liver disease such as alcoholic liver disease, autoimmune or viral hepatitis, drug-induced liver disease and primary biliary cirrhosis were excluded from the study. A retrospective review of their medical records was conducted along with histological reassessment of liver biopsies. Patients were histologically graded into four groups including non-NAFLD, NAFLD, NASH and cirrhosis. The cirrhotic group (C, $n=$ 26) was compared to non-cirrhotic group (NC, $n=84)$, which included patients with NASH, NAFLD and non-NAFLD.

\section{Procedure}

Primary bariatric surgical procedures, gastric banding (GB), sleeve gastrectomy (SG), Roux-en-Y gastric bypass (RNYGB) and biliopancreatic diversion with duodenal switch (BPD-DS) were included in the analysis. All patients underwent a laparoscopic approach for their bariatric surgery, except one who had a laparotomy and RNYGB. Three patients were abandoned due to unexpected intraoperative findings of portal hypertension, which we have considered as a contraindication to bariatric surgery. All these patients were included in the study on intention-to-treat analysis basis. All patients had preoperative weight and diet control advice and were followed for a 2-year period after surgery. Postoperative diet, vitamin supplementation and exercise advice followed national guidelines as per British Obesity and Metabolic Surgery Society.

All intraoperative biopsies were performed using a Tru-Cut biopsy of the left lobe of the liver and fixed in $10 \%$ formal saline solution. Paraffin block sections were cut at intervals of $5 \mathrm{~mm}$. Sections were stained with haematoxylin and eosin and Sirius Red for histological analysis. All liver biopsies were recalled and examined by two blinded liver histopathologists. Liver biopsies were assessed according to NAFLD activity score (NAS) [14]. Steatosis was graded into $0(<5 \%), 1(5-$ $33 \%), 2$ (34-66\%) and 3 (>60\%); hepatocyte ballooning was graded as 0 (none), 1 (few) and 2 (many); inflammation was graded as 0 (none), 1 ( $1-2$ foci per $\times 20$ field) and 2 ( $2-4$ foci per $\times 20$ field). This gave a total NAS of $0-8$. The presence of steatosis and hepatocyte ballooning was considered diagnostic of NASH. Fibrosis was graded as 0 (no fibrosis), 1a (zone 3 mild perisinusoidal fibrosis), $1 \mathrm{~b}$ (zone 3 moderate perisinusoidal fibrosis), 1c (periportal/portal fibrosis only), 2 (zone $3+$ periportal/portal fibrosis), 3 (bridging fibrosis) and 4 (cirrhosis). Fibrosis grade 3 and/or 4 was considered as advanced fibrosis. NASH fibrosis stage 4 was classed as cirrhosis.

\section{Data Collection}

Patient demographics (age, gender and BMI), comorbidities (diabetes, hypertension, obstructive sleep apnoea and metabolic syndrome), serum biochemistry (lipid profile, liver and renal functions), model for end-stage liver disease (MELD) and Child-Pugh scoring were recorded and used to assess 
patient's preoperative risk factors. Preoperative evidence of metabolic syndrome was recorded using the International Diabetes Federation consensus definition of the metabolic syndrome.

Diagnosis of diabetes, hypertension and hyperlipidaemia was confirmed from medical records. Diabetes was diagnosed if there had been use of insulin or oral hypoglycaemic agents, haemoglobin A1c level of greater than $6.5 \%$ or fasting blood glucose level of greater than $126 \mathrm{mg} / \mathrm{dl}$. Hypertension was defined as a sustained blood pressure of greater than 140/ $90 \mathrm{mmHg}$ or greater than $130 / 80 \mathrm{mmHg}$ for patients with diabetes or the use of antihypertensive medications. Dyslipidaemia was defined as the use of lipid-lowering therapy or on the basis of laboratory findings of low-density lipoprotein level of $130 \mathrm{mg} / \mathrm{dl}$ or more $(3.3 \mathrm{mmol} / \mathrm{l})$, triglyceride level of $150 \mathrm{mg} / \mathrm{ml}$ or more $(1.7 \mathrm{mmol} / \mathrm{l})$ or high-density lipoprotein level of less than $40 \mathrm{mg} / \mathrm{dl}(1.0 \mathrm{mmol} / \mathrm{l})$ in men and less than $50 \mathrm{mg} / \mathrm{dl}(1.3 \mathrm{mmol} / \mathrm{l})$ in women.

MELD score was calculated using preoperative biochemistry [bilirubin $(\mathrm{mg} / \mathrm{dl})$, serum sodium $(\mathrm{mEq} / \mathrm{l})$, international normalised ratio (INR), serum creatinine ( $\mathrm{mg} / \mathrm{dl})]$ and age. Child-Pugh class was calculated using the perioperative presence of ascites, hepatic encephalopathy and serum biochemistry [bilirubin $(\mathrm{mg} / \mathrm{dl})$, serum albumin $(\mathrm{g} / \mathrm{dl})$, prothrombin time (seconds)].

Clinical outcomes including 30-day postoperative complications, mortality and readmission rate as well as patient's length of stay were recorded. Postoperative surgical complications were graded according to the Clavien-Dindo classification (grades I-V) (Table 1) [15].

The $\mathrm{C}$ group was further sub-analysed for the presence of long-term liver-related complications such as mortality, decompensation, development of ascites, portal hypertension and MELD scores after surgery. We compared preoperative and postoperative MELD scores to study the impact of bariatric surgery on liver disease in the long term. Long-term follow-up

Table 1 Simplified description of the Clavien-Dindo classification [15]

\begin{tabular}{cl}
\hline Grades & Definition \\
\hline Grade I & $\begin{array}{c}\text { Any deviation from the normal postoperative course } \\
\text { without the need for pharmacological treatment } \\
\text { Requiring pharmacological treatment with drugs } \\
\text { other than for grade I complications }\end{array}$ \\
Grade II & $\begin{array}{c}\text { Requiring surgical, endoscopic or radiological intervention } \\
\text { Grade III }\end{array}$ \\
III a & $\begin{array}{l}\text { Intervention not under general anaesthesia } \\
\text { III b }\end{array}$ \\
Grade IV & $\begin{array}{c}\text { Life-threatening complication requiring IC/ICU } \\
\text { management }\end{array}$ \\
IV a & $\begin{array}{c}\text { Single organ dysfunction (including dialysis) } \\
\text { IV b }\end{array}$ \\
Multiorgan dysfunction \\
Grade V & Death of a patient \\
\hline
\end{tabular}

was conducted by retrospective analysis of available medical records and up-to-date information from general practitioners.

\section{Statistical Analysis}

The continuous variables are expressed as median (range), and categorical variables are expressed as percentage ( $n /$ total number) in order of C vs NC. Variables were assessed for normality using histograms and Q-Q plots. Chi-square test was applied to compare categorical variables. The Mann-Whitney $U$ test was used for non-parametric data analysis. Statistical analyses were performed using SPSS statistical package (version 23; IBM Corp., Armonk, NY, USA), and a $p$ value of less than 0.05 was considered statistically significant.

\section{Results}

Bariatric surgical procedures and liver biopsies were undertaken in a cohort of 110 patients. The median age was 45 years (24-75) and BMI $51 \mathrm{~kg} / \mathrm{m}^{2}$ (37-81). Three quarters of the cohort were female. Comorbidities included type 2 diabetes $(45 \%)$ and hypertension (52\%). Metabolic syndrome was present in $64 \%$ of patients. Operations performed included laparoscopic RNYGB (58\%, 64/110), laparoscopic sleeve gastrectomy (LSG) $(35 \%, 38 / 110)$, laparoscopic adjustable gastric banding (LABG) $(3 \%, 3 / 110)$ and laparoscopic biliopancreatic diversion with duodenal switch (DS) (2\%, 2/110). Three patients were abandoned after an unexpected intraoperative finding of portal hypertension. There was no 30-day perioperative mortality or postoperative liver decompensation. The overall complication rate was $22 \%$ (24/110), with $8 \%(9 / 110)$ of patients being suffered from ClavienDindo (CD) class $\geq$ III complications.

\section{Histological Grading}

Based on histological confirmation, our studied cohort consisted of 24\% (26/110) of cirrhotic patients, which were compared against a group of non-cirrhotic patients. The latter group consisted of $44 \%$ of patients with NASH (49/110), $22 \%$ (24/110) of patients with NAFLD and $10 \%$ (11/110) of patients with non-NAFLD. One histology showed advanced fibrosis grade 3 and had cirrhotic macro-nodular appearance of liver intraoperatively, another was later found to have portal hypertension and, therefore, 24\% (26/110) patients were classed as cirrhotic.

\section{Cirrhosis vs Non-cirrhosis Group (Table 2)}

The $\mathrm{C}$ group was older (years) ( 52 vs $43, p<0.05$ ), with lower BMI $\left(\mathrm{kg} / \mathrm{m}^{2}\right)(46 \mathrm{vs} 52, p<0.05)$ and less weight $(\mathrm{kg})(126 \mathrm{vs}$ $145, p<0.05)$, and was more likely to be of male gender (14/ 
Table 2 Comparison of parameters between cirrhosis and non-cirrhosis groups

\begin{tabular}{lllll}
\hline Patient characteristics & Overall & Cirrhosis (C) & Non-cirrhosis (NC) & $p$ value \\
\hline Number of patients & 110 & 26 & 84 & $<0.001^{*}$ \\
Age (years) & $45(24-75)$ & $52(37-66)$ & $43(24-75)$ & $0.001^{*}$ \\
Sex (male/female) & $25 / 74$ & $14 / 12$ & $15 / 69$ & $<0.05^{*}$ \\
BMI $\left(\mathrm{kg} / \mathrm{m}^{2}\right)$ & $51(37-81)$ & $46(38-63)$ & $52(37-81)$ & $<0.01^{*}$ \\
Weight $(\mathrm{kg})$ & $142(90-222)$ & $126(90-203)$ & $145(97-222)$ & $<0.001^{*}$ \\
T2DM & $50(45.4 \%)$ & $22(84 \%)$ & $28(33 \%)$ & $<0.005^{*}$ \\
Metabolic syndrome & $70(63.6 \%)$ & $25(96 \%)$ & $45(53.5 \%)$ & $\mathrm{NS}$ \\
Hypertension & $57(51.8 \%)$ & $17(65 \%)$ & $40(47.6 \%)$ & $\mathrm{NS}$ \\
OSA & $59(53.6 \%)$ & $14(53.8 \%)$ & $45(53.5 \%)$ & $\mathrm{NS}$ \\
Procedures & & & $2(2.3 \%)$ & \\
LGB & $3(2.7 \%)$ & $1(3.85 \%)$ & $31(36.9 \%)$ & \\
LSG & $38(34.5 \%)$ & $7(26.9 \%)$ & $49(58.3 \%)$ & \\
LRNYGB & $64(58.2 \%)$ & $14(53.8 \%)$ & $0(0 \%)$ & $\mathrm{NS}$ \\
RNYGB & $0(0 \%)$ & $1(3.8 \%)$ & $4(2.4 \%)$ & $<0.05^{*}$ \\
LDS & $2(1.82 \%)$ & $0(0 \%)$ & $14(16.7 \%)$ & \\
LOS (days) & $4(1-51)$ & $5(2-20)$ & $10(38.5 \%)$ & \\
30-day complications & $24(21.8 \%)$ & &
\end{tabular}

Asterisk indicates significant values. NS indicates non-significant outcomes. Continuous data is expressed as median (range). Categorical data is presented as total number $(n)$ and percentage (\%). Categorical data was compared with chi-square test, and continuous data was compared with the Mann-Whitney $U$ test

$T 2 D M$ type 2 diabetes mellitus, $L G B$ laparoscopic gastric band, $L S G$ laparoscopic sleeve gastrectomy, $L R N Y G B$ laparoscopic Roux-en-Y gastric bypass, $L D S$ laparoscopic biliopancreatic diversion and duodenal switch, $L O S$ length of stay
26 vs 15/84, $p<0.01$ ) than the NC group. Type 2 diabetes mellitus and metabolic syndrome were significantly more common in the $\mathrm{C}$ group $(22 / 26$ vs $28 / 84, p<0.001 ; 25 / 26$ vs $45 / 84, p<0.001$, respectively). Hypertension and obstructive sleep apnoea were similar in both groups (Table 2).

The C group had significantly higher levels of gammaglutamyl transferase (GGT, U/l) (119, 29-450 vs 37, 8-321, $p<0.05)$, aspartate aminotransferase (AST, U/l) $(47,18-384$ vs $22,13-83, p<0.05)$, prothrombin time (INR) $(1.07,0.8-1.4$ vs $1,0.8-1.2, p<0.05)$ and lower platelets $\left(\times 10^{9} / 1\right)(221,59-892$ vs $294,198-505, p<0.05)$ compared to the NC group. There were no significant differences between bilirubin, alkaline phosphatase, serum albumin, creatinine, cholesterol and triglycerides. The $\mathrm{C}$ group had a median MELD score of 7 (6-24), and majority were Child-Pugh A (99\%, 104/105).

The overall rate of complications was greater in the $\mathrm{C}$ group $(10 / 26$ vs $14 / 84, p<0.05)$ than in the $\mathrm{NC}$ group. The $\mathrm{CD}$ class $\geq$ III complication rate was $12 \%(3 / 26)$ in the $\mathrm{C}$ group compared to $7 \%(6 / 84)$ in the NC group $(p<0.05)$ (Tables 3 and 4). In sub-group analysis, the $\mathrm{C}$ group had significantly higher rate and severity of complications compared to the other groups (NASH, NAFLD, non-NAFLD) $(p<0.05)$. The overall complication rate in the NASH subgroup was $22 \%$ (11/49), while the rate of CD class $\geq$ III complication was $8 \%(4 / 49)$. The CD class $\geq$ III complication rate in the other subgroups was as follows: for NAFLD, $8 \%(2 / 24)$, and for non-NAFLD, $0 \%(0 / 11)$.

The $\mathrm{C}$ group was further followed up for a median of 4.5 years (range 2-11 years) to monitor long-term cirrhotic complications and the effect of bariatric surgery on overall mortality. None of the patients developed cirrhosis-related complications such as decompensation, ascites or portal hypertension, and there was no mortality.

There was significant improvement in postoperative MELD scores (7 vs 6, $p$ value $<0.01$ ) at a 4.5-year followup (range 2-11 years).

Complication rates were not influenced by histological liver grading; advanced fibrosis (grades 3 and 4) had a similar rate of complication with no or mild fibrosis (11/47 vs 13/63). Similarly, the degree of inflammation, whether advanced (grade 2) or less, did not influence the complication rate [21\% (5/24) vs $22 \%(19 / 86)]$. The severity of complications was also not influenced by an increasing degree of fibrosis or inflammation. The overall length of stay and rate of readmission were similar in both groups.

\section{Discussion}

Morbid obesity is associated NAFLD, with the development of metabolic diseases, cardiovascular disease and decreased 
Table 3 Comparison of severity of complication between the cirrhosis group and non-cirrhosis group according to the ClavienDindo classification $(p$ value $<$ 0.05)

\begin{tabular}{llll}
\hline CD grade of complication & Overall, $n(\%$ age $)$ & Cirrhosis $(\mathrm{C}), n(\%$ age $)$ & Non-cirrhosis (NC), $n(\%$ age $)$ \\
\hline I & $2(1.8)$ & $1(3.8)$ & $1(1.19)$ \\
II & $13(11.8)$ & $6(23)$ & $7(8.3)$ \\
IIIA & $0(0)$ & $0(0)$ & $0(0)$ \\
IIIB & $6(5.4)$ & $1(3.8)$ & $5(5.9)$ \\
IVA & $1(0.9)$ & $0(0)$ & $1(1.1)$ \\
IVB & $2(1.8)$ & $2(7.7)$ & $0(0)$ \\
\hline
\end{tabular}

The difference in severity was assessed using chi-square test. The cirrhosis group had more severe complications longevity [16-18]. An elevated BMI in a cirrhotic patient is considered to be a strong predictor of decompensation [19]. Decreasing weight in the latter group may thus improve their long-term outcome by reducing the risk of decompensation, improve eligibility for liver transplantation as well as resolution of comorbidities and decrease the risk of surgery [11, 20, 21].

Diet and exercise have an important role in the treatment of obesity. Their effectiveness in NAFLD has been demonstrated, with improvement in liver enzymes and liver morphology even in the absence of weight loss [22-24]. In obese cirrhotic patients, there is a lack of randomised evidence in the evaluation of diet or exercise. Pharmacological measures have a role in the management of obesity, with total body weight loss of up to $10 \%$. They are not as effective as surgery, and there is rebound weight gain once medication is discontinued.

Surgery in cirrhotic patients is known to be associated with an increased perioperative risk for abdominal and nonabdominal surgery, due to multiple risk factors such as hypoalbuminemia, anaemia, malnutrition, coagulation abnormalities, thrombocytopenia, renal impairment, susceptibility to infections and risk of decompensation [10,12]. Postoperatively, these patients may develop liver dysfunction requiring intensive monitoring, ascites which may leak through the wound or compromise healing of anastomosis and life-threatening multiple organ failure. In addition, thickening of the peritoneum, splenomegaly and presence of varices may impose technical difficulty and increase the risk of bleeding [25].

Type 2 diabetes mellitus and metabolic syndrome were significantly more common in the $\mathrm{C}$ group compared to the $\mathrm{NC}$ group [ $84 \%$ vs $33 \%(p<0.001)$ and $96 \%$ vs $53 \%$ $(p<0.001)$, respectively]. Metabolic syndrome is known to adversely affect the perioperative outcome of various other surgical procedures, but its effects on postoperative complications after bariatric surgery have not been studied in detail. In a recent study, involving 168,501 bariatric patients and 18,516 metabolic syndrome patients (11\%), Gondal et al. [26] suggested that the presence of metabolic syndrome does not predict postoperative morbidity or complications in bariatric patients. However, the effects of metabolic syndrome on the outcome of bariatric surgery in cirrhotic patients have yet to be established.

The cirrhotic patients in this cohort undergoing bariatric surgery had a higher complication rate (38\%) and severity of complication compared to those in the non-cirrhotic group (16\%). Similar complication rates of $30-35 \%$ have been found by other investigators performing bariatric surgery in cirrhotic patients $[12,20,27]$. The severity of complication of CD class $\geq$ III was $12 \%$ in cirrhotic patients compared to $7 \%$

Table 4 Details of postoperative complications (Clavien-Dindo class $\geq$ III) along with the type of surgery in cirrhotic and non-cirrhotic patients

\begin{tabular}{|c|c|c|c|}
\hline Histology & Type of surgery & Complication & Intervention \\
\hline \multirow[t]{3}{*}{ Cirrhosis (C) group } & Open SG & Hematemesis & OGD — no bleeding source found \\
\hline & LRYNGB & $\begin{array}{l}\text { Type } 1 \text { respiratory failure pulmonary } \\
\text { oedema and HAP }\end{array}$ & Transfer to ITU for inotropic support \\
\hline & LRYNGB & Post-op hypoventilation, bleeding from port site & ITU stay for 3 days for respiratory support \\
\hline \multirow{6}{*}{$\begin{array}{l}\text { Non-cirrhosis (NC) } \\
\text { group }\end{array}$} & LSG & Dysphagia & Laparoscopy and division of suture over staple \\
\hline & LSG & Readmission abdominal pain & Diagnostic laparoscopy—no cause found \\
\hline & LSG & Postoperative gastric leak & $\begin{array}{l}\text { Laparoscopy, washout of peritoneal cavity and } \\
\text { omental patch }\end{array}$ \\
\hline & LRYNGB & Readmission with severe abdominal pain & $\begin{array}{l}\text { Laparoscopy and repair of perforated duodenal } \\
\text { ulcer (omental patch) }\end{array}$ \\
\hline & LSG & Slow to progress, persistent vomiting & NJ tube insertion for feeding \\
\hline & LRYNGB & $\begin{array}{l}\text { Postoperative rhabdomyolysis and } \\
\text { acute kidney injury }\end{array}$ & Transfer to liver ITU—symptomatic treatment \\
\hline
\end{tabular}

$O G D$ orogastroduodenoscopy, HAP hospital-acquired pneumonia, ITU intensive treatment unit, $N J$ nasojejunal 
Table 5 Comparison of the type of surgery and Clavien-Dindo (CD) classification complication rate between the $\mathrm{C}$ and $\mathrm{NC}$ groups $(p=\mathrm{NS})$

\begin{tabular}{|c|c|c|c|c|}
\hline \multirow[t]{2}{*}{ CD complications } & \multicolumn{2}{|c|}{ Cirrhosis (C) group, $n$ (\%age) } & \multicolumn{2}{|c|}{ Non-cirrhosis (NC) group, $n$ (\%age) } \\
\hline & LRNYGB & LSG & LRNYGB & LSG \\
\hline I & $1(6.6)$ & & & $1(3)$ \\
\hline II & $4(26.6)$ & $1(14)$ & $4(8.16)$ & $3(9.6)$ \\
\hline \multicolumn{5}{|l|}{ IIIA } \\
\hline IIIB & & $1(14)$ & $1(2)$ & $4(12.9)$ \\
\hline IVA & & & $1(2)$ & \\
\hline IVB & $2(13.3)$ & & & \\
\hline Total & $7(46.6)$ & $2(28.6)$ & $6(12)$ & $8(25.8)$ \\
\hline
\end{tabular}

Laparoscopic gastric band and laparoscopic duodenal switch did not have any complications. The percentage is calculated by dividing the number of patients in one Clavien-Dindo class with the total number of procedurerelated complication

$L R N Y G B$ laparoscopic Roux-en-Y gastric bypass, $L S G$ laparoscopic sleeve gastrectomy in non-cirrhotic patients $(p<0.05)$. The overall median length of stay was similar between the cirrhotic and non-cirrhotic groups [5 (2-20) vs $4(1-51), p=\mathrm{NS}]$, and this was despite the increased complication rate in the cirrhotic group. No perioperative mortality was observed in either group.

In-hospital mortality is shown to be $1.2 \%$ for all cirrhotic patients compared to $0.9 \%$ in compensated cirrhotic patients and $0.3 \%$ in non-cirrhotic patients [1]. Our study shows that in well-compensated cirrhotic bariatric patients (Child-Pugh A), various forms of bariatric surgical procedures are feasible at acceptable complication rate. In addition, short- and long-term perioperative mortality is rare (0/26 in our study). Rebibo et al. [28] and Woodford et al. [29] report on safety of LSG and LGB on similar patients with cirrhosis and confirm $0 \%$ perioperative mortality, respectively. The overall complication rate of $22 \%$ in our study is skewed by the number of cirrhotic patients in the overall cohort, and possibly the BMI (median $51 \mathrm{~kg} / \mathrm{m}^{2}$, range 37-81). The incidence of complications in super-obese patients $\left(\mathrm{BMI}>50 \mathrm{~kg} / \mathrm{m}^{2}\right.$ ) has been reported to be fourfold higher compared to the morbidly obese group $\left(\mathrm{BMI}<50 \mathrm{~kg} / \mathrm{m}^{2}\right)$. Eighty percent of the deaths following bariatric surgery occur in the super-obese patients [30-32]. Benotti et al. [33] confirmed that BMI $>50 \mathrm{~kg} / \mathrm{m}^{2}$ is the strongest predictor of mortality in a cohort of 185,315 patients undergoing RNYGB.

Evidence regarding safety of bariatric surgery as well as type of optimal procedure is lacking in patients with advanced fibrosis or cirrhosis, and more evidence is required to assess whether an increased risk of complications in such patients is truly related to the degree of liver fibrosis and/or inflammation [30]. In our study, the $\mathrm{C}$ group had significantly higher rate and severity of complication as compared to the NC group. There was no associated correlation between the type of surgery and complication rate in our small cirrhotic cohort (Table 5).

Jan et al. [11], in their pooled data of nine similar studies with an overall complication rate of $22 \%$, have observed lower complication rate in cirrhotic patients undergoing LSG and/or LGB when compared to more radical malabsorptive bariatric procedures including RYNGB and BPD [11]. However, these differences could not be substantiated statistically, and therefore, safety and long-term risks and benefits of each type of surgical procedure cannot be confirmed.

In our cirrhotic cohort of patients, none of the patients develop long-term postoperative cirrhosis-related complications such as ascites, decompensation or development of portal hypertension at a long-term median follow-up of 4.5 years (range 2-11 years). Comparison of preoperative and postoperative MELD score in cirrhotic patients revealed significant improvement ( 7 vs $6, p<0.02$ ), and this may suggest potential benefit of undergoing bariatric surgery in morbidly obese cirrhotic patients.

The risk of morbidity and mortality after bariatric surgery in cirrhotic well-compensated Child-Pugh A patients is further increased by the presence of portal hypertension. The latter is a recognised contraindication to operative intervention in bariatric surgery [34]. In our cohort, three patients had intraoperative signs of portal hypertension, and therefore, we had to abandon their procedures. There is emerging data that bariatric surgery may be feasible in cirrhotic patients with mild portal hypertension with preoperative placement of transjugular intrahepatic portosystemic shunt (TIPS) [34]. Hanipah et al. [35] have performed LSG and RNYGB in a tertiary academic centre in 13 cirrhotic patients with portal hypertension. They have reported a $23 \%$ 30-day complication rate with no mortalities [36]. Six of 13 patients had either preoperative or postoperative TIPS insertion. Shimizu et al. [12] successfully performed LSG in 2 patients following preoperative TIPS placement.

One of the strengths of this study is that all patients were operated on by one primary surgeon and thus reducing operator bias. Limitations include study's retrospective nature, small number of patients in each group and, therefore, risk 
of type II error. In the future, studies with larger cohort and matched comparative groups would create a more robust methodology. Performing routine intraoperative liver biopsy in bariatric patients would promote learning of liver pathologies and outcomes in such patients.

This study does confirm a twofold increase in the complication rate in cirrhotic compared to non-cirrhotic patients undergoing bariatric surgery. However, with appropriate counselling, preoperative assessment and medical support, it is feasible to undergo bariatric surgery safely. Surgery for the cirrhotic patient, especially with the presence of portal hypertension, should be performed in tertiary centres with specialist liver support.

Our results show that although bariatric surgery carries a relatively higher risk of complications in immediate postoperative period (30 days), long-term cirrhosis-related complications (> 52 weeks) and mortality are not influenced in this small cohort with long-term follow-up. The benefits of weight loss and reversal of comorbidities may potentially improve liver function. Bariatric surgery may be considered in compensated cirrhotic patients in specialist centres to improve long-term disease outcome.

\section{Compliance with Ethical Standards}

Conflict of Interest The authors declare that they have no conflict of interest.

Ethical Approval All procedures performed in this study were in accordance with the ethical standards of the institutional and/or national research committee and with the 1964 Helsinki Declaration and its later amendments or comparable ethical standards.

Consent Statement For this type of study, formal consent is not required.

All authors are in agreement with the content of the article.

Open Access This article is distributed under the terms of the Creative Commons Attribution 4.0 International License (http:// creativecommons.org/licenses/by/4.0/), which permits unrestricted use, distribution, and reproduction in any medium, provided you give appropriate credit to the original author(s) and the source, provide a link to the Creative Commons license, and indicate if changes were made.

\section{References}

1. Andersen T, Gluud C. Liver morphology in morbid obesity: a literature survey. Int J Obes. 1984;8(2):97-16.

2. Gentile CL, Pagliassotti MJ. The role of fatty acids in the development and progression of nonalcoholic fatty liver disease. J Nutr Biochem. 2008;19(9):567-76.

3. Day CP. From fat to inflammation. Gastroenterology. 2006;130(1): 207-21.

4. Jou J, Choi SS, Diehl AM. Mechanisms of disease progression in nonalcoholic fatty liver disease. Semin Liver Dis. 2008;28(4):370-9.
5. Dowman JK, Tomlinson JW, Newsome PN. Pathogenesis of nonalcoholic fatty liver disease. QJM. 2010;103(2):71-83.

6. Svegliati-Baroni G, De Minicis S, Marzioni M. Hepatic fibrogenesis in response to chronic liver injury: novel insights on the role of cell-to-cell interaction and transition. Liver Int. 2008;28(8):152-64.

7. Xia JL, Dai C, Michalopoulos GK, et al. Hepatocyte growth factor attenuates liver fibrosis induced by bile duct ligation. Am J Pathol. 2006;168(5):1500-12.

8. Ranlov I, Hardt F. Regression of liver steatosis following gastroplasty or gastric bypass for morbid obesity. Digestion. 1990;47(4):208-14.

9. Chang SH, Stoll CR, Song J, et al. The effectiveness and risks of bariatric surgery: an updated systematic review and meta-analysis, 2003-2012. JAMA Surg. 2014;149(3):275-87.

10. Kral JG, Thung SN, Biron S, et al. Effects of surgical treatment of the metabolic syndrome on liver fibrosis and cirrhosis. Surgery. 2004; 135(1):48-58.

11. Jan A, Narwaria M, Mahawar K, et al. A systematic review of bariatric surgery in patients with liver cirrhosis. Obes Surg. 2015;25(8):1518-26.

12. Shimizu H, Phuong V, Maia M, et al. Bariatric surgery in patients with liver cirrhosis. Surg Obes Relat Dis. 2013;9(1):1-6.

13. Mosko JD, Nguyen GC. Increased perioperative mortality following bariatric surgery among patients with cirrhosis. Clin Gastroenterol Hepatol. 2011;9(10):897-901.

14. Kleiner DE, Brunt EM, Van Natta M, et al. Design and validation of a histological scoring system for nonalcoholic fatty liver disease. Hepatology. 2005;41(6):1313-21.

15. Dindo D, Demartines N, Clavien PA. Classification of surgical complications: a new proposal with evaluation in a cohort of 6336 patients and results of a survey. Ann Surg. 2004;240(2): 205-13.

16. Vuppalanchi R, Chalasani N. Non-alcoholic fatty liver disease and non-alcoholic steatohepatitis: selected practical issues in their evaluation and management. Hepatology. 2009;49(1):306-17.

17. Ong JP, Pitts A, Younossi ZM. Increased overall mortality and liver related mortality in non-alcoholic fatty liver disease. J Hepatol. 2008;49(4):608-12.

18. Adams LA, Lymp JFS, Sauver J, et al. The natural history of nonalcoholic fatty liver disease: a population-based cohort study. Gastroenterology. 2005;129(1):113-21.

19. Berzigotti A, Garcia-Tsao G, Bosch J, et al. Obesity is an independent risk factor for clinical decompensation in patients with cirrhosis. Hepatology. 2011;54(2):555-61.

20. Takata MC, Campos GM, Ciovica R, et al. Laparoscopic bariatric surgery improves candidacy in morbidly obese patients awaiting transplantation. Surg Obes Relat Dis. 2008;4(2):159-64.

21. Chen Y, Wang X, Wang J, et al. Excess body weight and the risk of primary liver cancer: an updated meta-analysis of prospective studies. Eur J Cancer. 2012;48(14):2137-45.

22. Clark JM. Weight loss as a treatment for non-alcoholic fatty liver disease. J Clin Gastroenterol. 2006;40(Suppl 1):S39-43.

23. Cave M, Deaciuc I, Mendez C, et al. Nonalcoholic fatty liver disease: predisposing factors and the role of nutrition. J Nutr Biochem. 2007;18(3):184-95.

24. Nobili V, Alisi A, Raponi M. Pediatric non-alcoholic fatty liver disease: preventive and therapeutic value of lifestyle intervention. World J Gastroenterol. 2009;15(48):6017-22.

25. Peck-Radosavljevic M, Angeli P, Cordoba J, et al. Managing complications in cirrhotic patients. United European Gastroenterol J. 2015;3(1):80-94.

26. Gondal A. B. et al. Metabolic syndrome and short-term postoperative complications in bariatric surgery: the whole is not always greater than the sum of its parts. J Am Coll Surg. 2018;227(4):72-3. 
27. Dallal RM, Mattar SG, Lord JL, et al. Results of laparoscopic gastric bypass in patients with cirrhosis. Obes Surg. 2004;14(1):47-53.

28. Rebibo L, Gerin O, Verhaeghe P, et al. Laparoscopic sleeve gastrectomy in patients with NASH-related cirrhosis: a case-matched study. Surg Obes Relat Dis. 2014;10(3):405-10.

29. Woodford RM, Burton PR, O'Brien PE, et al. Laparoscopic adjustable gastric banding in patients with unexpected cirrhosis: safety and outcomes. Obes Surg. 2015;25(10):1858-62.

30. Van Steenbergen W, Lenchmans S. Liver disturbances in obesity and diabetes mellitus. Int J Obes. 1995;199(Suppl 3):S27-36.

31. Pinho S, Carvalho M, Soares M, et al. Obesity surgery mortality risk score: can we go beyond mortality prediction? J Anesth Clin Res. 2015;6(9):562.

32. Santo MA, Riccioppo D, Pajecki D, et al. Preoperative weight loss in super-obese patients: study of the rate of weight loss and its effects on surgical morbidity. Clinics. 2014;69(12):828-34.
33. Benotti P, Wood GC, Winegar DA, et al. Risk factors associated with mortality after Roux-en-Y gastric bypass surgery. Ann Surg. 2014;259(1):123-30.

34. Pestana L, Swain J, Dierkhising R, et al. Bariatric surgery in patients with cirrhosis with and without portal hypertension: a singlecenter experience. Mayo Clin Proc. 2015;90(2):209-15.

35. Hanipah ZN, Punchai S, McCullough A, et al. Bariatric surgery in patients with cirrhosis and portal hypertension. Obes Surg. 2018;28(11):3431-8.

36. Miñambres I, Rubio MA, de Hollanda A, et al. Outcomes of bariatric surgery in patients with cirrhosis. Obes Surg. 2018; https:// doi.org/10.1007/s11695-018-3562-8.

Publisher's Note Springer Nature remains neutral with regard to jurisdictional claims in published maps and institutional affiliations. 